\title{
AN ISOPERIMETRIC INEQUALITY INVOLVING CONFORMAL MAPPING
}

\author{
ANDREW ACKER \\ Abstract. An isoperimetric inequality due to $\mathrm{L}$. Bieberbach is generalized \\ to the case of weighted areas.
}

1. Introduction. L. Bieberbach [4] showed in 1913 that of all regions $\Omega \subset \mathbf{C}$ which are images of the disc $|z|<r$ under conformal mappings $F(z)$ normalized by the condition $\left|F^{\prime}(0)\right|=1$, the discs $\left|z-z_{0}\right|<r, z_{0} \in \mathbf{C}$, have the least area. The analogous isoperimetric inequality for doubly-connected regions (in 2 dimensions) was obtained in 1917 by T. Carleman [5], who showed that of all doubly-connected regions of area $\pi\left(r_{1}^{2}-r_{0}^{2}\right)>0$, each of which encircles a region of area $\pi r_{0}^{2}$, the annuli $r_{0}<\left|z-z_{0}\right|<r_{1}, z_{0} \in \mathbf{C}$, have the smallest capacitance. A 3-dimensional generalization of Carleman's result was obtained by G. Szegö [7]. An interesting survey of the large number of isoperimetric inequalities which have been found in the meantime is given in [6].

In [1], the author has extended Carleman's result to a class of capacitanceminimization problems involving doubly-connected regions with specified weighted area and a fixed inner boundary component. For convenience, this result is stated in Theorem 1 below. The prupose of this note is to prove (by application of Theorem 1) an analogous generalization of Bieberbach's minimization principle to the case of weighted areas. This result is Theorem 2.

In the statement of Theorem 1 which follows, $\Omega \subset R^{2}$ is a doubly-connected region with the Jordan curves $\Gamma^{*}$ and $\Gamma$ as inner and outer boundary components. The capacitance of $\Omega$ (which was called the heat flow in [1]) is defined by $K=\int_{\gamma}|\nabla U(p)| \cdot|d p|$, where $U(p)$ is the harmonic measure of $\Gamma^{* 1}$ in $\Omega$ and $\gamma$ is an equipotential curve of $U(p)$ in $\Omega . \bar{\Omega}, \bar{\Gamma}, \bar{\Gamma}^{*}, \bar{U}(p)$, and $\bar{K}$ refer to a second region with the same properties.

THEOREM 1. Let $a(p)>0$ be a continuous real function on $R^{2}$ such that $\lambda \cdot a(\lambda p)$ is monotone nondecreasing in $\lambda \in(0, \infty)$ for each $p \in R^{2}$, and let the Jordan curve $\Gamma^{*}$ be the graph in polar coordinates of a continuous function $r^{*}(\theta)>0,0<\theta \leqslant 2 \pi$. Then:

Presented to the Society, September 30, 1976; received by the editors October 1, 1976.

AMS (MOS) subject classifications (1970). Primary 30A40, 30A42, 30A44, 31A15.

Key words and phrases. Isoperimetric inequality, free boundary optimization.

'The harmonic measure of $\Gamma^{*}$ in $\Omega$ is a harmonic function $U(p)$ on $\Omega$, continuous on Closure( $(\Omega)$, such that $U(p)=1$ on $\Gamma^{*}$ and $U(p)=0$ on $\Gamma$. 
(a) There exists a doubly-connected region $\Omega$ (having $\Gamma^{*}$ as its inner boundary component) such that $|\nabla U(p)|$ has a continuous extension to $\Omega \cup \Gamma$ satisfying $|\nabla U(p)|=a(p)$ on $\Gamma$.

(b) If $\bar{\Omega} \neq \Omega$, but $\bar{\Gamma}^{*}=\Gamma^{*}$, then

$$
\bar{K}+|\bar{\Omega}|>K+|\Omega|,
$$

where $|\Omega|$ and $|\bar{\Omega}|$ are weighted areas defined by $|\Omega|=\iint_{\Omega} a^{2}(p) d x d y$. Thus, $\Omega$ uniquely minimizes $\bar{K}$ in the class of regions $\bar{\Omega}$ with $\bar{\Gamma}^{*}=\Gamma^{*}$ and $|\bar{\Omega}|<|\Omega|$, and uniquely minimizes $|\bar{\Omega}|$ in the class of regions $\bar{\Omega}$ with $\bar{\Gamma}^{*}=\Gamma^{*}$ and $\bar{K}<K$. (See [1, Theorems 10, 11 and 12].)

In the statement of Theorem $2, \Omega$ represents any simply-connected region in $\mathbf{C}$ which contains $\mathbf{O}$ (the origin), is bounded by a Jordan curve $\Gamma$, and is conformally mapped onto the unit disc $|z|<1$ by a function $F(z)$ satisfying $F(0)=0$. The notation $\bar{\Omega}, \bar{\Gamma}, \bar{F}(z)$ refers to a second region with the same properties.

THEOREM 2. Let a continuous real function $a(z)>0$ on $\mathrm{C}$ and a region $\Omega \subset \mathbf{C}$ be chosen such that:

(a) $\Gamma$ is the graph in polar coordinates of a continuous function $r(\theta)>0$, $0<\theta<2 \pi$,

(b) $\left|F^{\prime}(z)\right|$ has a continuous extension to $\Omega \cup \Gamma$ such that $\left|F^{\prime}(z)\right|=a(z)$ on $\Gamma$, and

(c) for each $z \in \Gamma, \lambda \cdot a(\lambda z) \leqslant a(z)$ for all $0<\lambda<1$ and $\lambda \cdot a(\lambda z)>a(z)$ for all $\lambda>1$. Then for any other region $\bar{\Omega}$ we have

$$
2 \pi \cdot \operatorname{Ln}\left(\left|\bar{F}^{\prime}(0)\right|\right)+|\bar{\Omega}| \geqslant 2 \pi \cdot \operatorname{Ln}\left(\left|F^{\prime}(0)\right|\right)+|\Omega|,
$$

where $|\Omega|$ and $|\bar{\Omega}|$ are weighted areas defined by $|\Omega|=\iint_{\Omega} a^{2}(z) d x d y$. Thus, $\Omega$ minimizes $\left|\bar{F}^{\prime}(0)\right|$ in the class of regions $\bar{\Omega}$ having $|\bar{\Omega}| \leqslant|\Omega|$ and minimizes $|\bar{\Omega}|$ in the class of regions $\bar{\Omega}$ having $\left|\bar{F}^{\prime}(0)\right| \leqslant\left|F^{\prime}(0)\right|$. If the inequalities in (c) are strict, then (2) can reduce to equality only when $\bar{\Omega}=\Omega$. In this case, $\Omega$ solves the above two minimum problems uniquely.

REMARK 3. For any region $\Omega$ whose boundary $\Gamma$ has bounded curvature and satisfies assumption (a), there are infinitely many functions $a(z)$ which satisfy conditions (b) and (c). The inequality (2) is strongest (for a given boundary $\Gamma$ ) when $a(z)$ is chosen such that the inequalities in (c) reduce identically to equalities. Actually $|\Omega|=|\bar{\Omega}|=\infty$ in this case due to the strong singularity in $a^{2}(z)$ at 0 , but the content of Theorem 2 is preserved by replacing $|\Omega|-|\bar{\Omega}|$ by $|\Omega \backslash \bar{\Omega}|-|\bar{\Omega} \backslash \Omega|$ in (2). If $\lambda \cdot a(\lambda z)$ is monotone nondecreasing in $\lambda \in(0, \infty)$ for each $z \in \mathbf{C}$, then it seems likely (by analogy to [1, Lemma 11]) that a unique region $\Omega$ exists such that conditions (a) and (b) (and (c) trivially) are fulfilled. However, this has apparently not been proved.

2. The proof of Theorem 2. It is more convenient to prove the following Theorem 4, which is the equivalent of Theorem 2 in the context of harmonic functions. In Theorem $4, \Omega_{i}, i=1,2$, is a simply-connected region in $R^{2}$ 
which contains 0 and is bounded by a Jordan curve $\Gamma_{i} \cdot W_{i}(p), i=1,2$, is a continuous function on $\Omega_{i} \cup \Gamma_{i}$ which is harmonic on $\Omega_{i}$ and satisfies the boundary condition $W_{i}(p)=(1 / 2 \pi) \cdot \operatorname{Ln}(|p|)$ on $\Gamma_{i}$. We define $U_{i}(p)=$ $-(1 / 2 \pi) \cdot \operatorname{Ln}(|p|)+W_{i}(p)$ on $\Omega_{i} \cup \Gamma_{i}, i=1,2$.

THEOREM 4. Let a continuous real function $a(p)>0$ on $R^{2}$ and a region $\Omega_{1} \subset R^{2}$ be chosen such that:

(a) $\Gamma_{1}$ is the graph in polar coordinates of a continuous function $r(\theta)>0$, $0 \leqslant \theta \leqslant 2 \pi$,

(b) $\left|\nabla U_{1}(p)\right|$ has a continuous extension to $\Omega_{1} \cup \Gamma_{1}$ such that $\left|\nabla U_{1}(p)\right|=$ $a(p)$ on $\Gamma_{1}$, and

(c) for each $p \in \Gamma_{1}, \lambda \cdot a(\lambda p) \leqslant a(p)$ for all $0 \leqslant \lambda<1$ and $\lambda \cdot a(\lambda p) \geqslant a(p)$ for all $\lambda>1$. Then for any other region $\Omega_{2}$ we have

$$
W_{2}(0)-\left|\Omega_{2}\right| \leqslant W_{1}(0)-\left|\Omega_{1}\right| \text {, }
$$

where $\left|\Omega_{i}\right|=\iint_{\Omega_{i}} a^{2}(p) d x d y, i=1$, 2. If the inequalities in (c) are strict, then (3) can reduce to equality only when $\Omega_{1}=\Omega_{2}$.

Proof. Since $\left|\Omega_{2}\right|-\left|\Omega_{1}\right|=\left|\Omega_{2} \backslash \Omega_{1}\right|-\left|\Omega_{1} \backslash \Omega_{2}\right|$, the inequality (3) can be rewritten:

$$
\left|\Omega_{2} \backslash \Omega_{1}\right|-\left|\Omega_{1} \backslash \Omega_{2}\right| \geqslant W_{2}(0)-W_{1}(0)
$$

Since $0 \in \Omega_{i}, i=1,2$, (4) continues to be meaningfull when the weight function $a^{2}(p)$ is singular at 0 . Define $a^{*}(p)$ on $R^{2} \backslash\{0\}$ such that $a^{*}(p)=\nu$ - $a(\nu p)$, where $\nu>0$ is chosen such that $\nu p \in \Gamma_{1}$. From assumption (c) we have that $0<a(p) \leqslant a^{*}(p)$ in $\Omega_{1} \backslash \Omega_{2}$ and $0<a^{*}(p) \leqslant a(p)$ in $\Omega_{2} \backslash \Omega_{1}$. Thus:

$$
\left|\Omega_{2} \backslash \Omega_{1}\right|-\left|\Omega_{1} \backslash \Omega_{2}\right| \geqslant\left|\Omega_{2} \backslash \Omega_{1}\right|^{*}-\left|\Omega_{1} \backslash \Omega_{2}\right|^{*},
$$

where || and ||$^{*}$ are respectively weighted areas defined by the weight functions $a^{2}(p)$ and $\left(a^{*}(p)\right)^{2}$. If the inequalities in (c) are strict, then (5) can reduce to an equality only when $\Omega_{1}=\Omega_{2}$. Thus, to prove Theorem 4 it suffices to show that (4) holds (under assumptions (a) and (b)) in the case where $a(p) \equiv a^{*}(p)$. To this end, we first make the additional assumption that $\Gamma_{1}$ and $\Gamma_{2}$ have bounded curvature. For $0<\delta<\delta_{0}:=\inf \left\{|p|: p \in \Gamma_{1}\right.$ $\left.\cup \Gamma_{2}\right\}$, and $i=1,2$, we define the doubly-connected regions $\Omega_{i, \delta}=\left\{p \in \Omega_{i}\right.$ : $|p|>\delta\}$ and the inner boundary component $\Gamma_{\delta}^{*}=\left\{p \in R^{2}:|p|=\delta\right\}$. The definitions of the harmonic measure $U_{i, \delta}(p)$ on $\Omega_{i, \delta}$ and the capacitance $K_{i, \delta}$ of $\Omega_{i, \delta}$ are analogous to those given preceding Theorem 1. Since $\Gamma_{1}$ has bounded curvature, $\left|\nabla U_{1, \delta}(p)\right|$ has a continuous extension to $\Omega_{1, \delta} \cup \Gamma_{1}$, and we can define $a_{\delta}(p)$ on $R^{2} \backslash\{0\}$ such that $a_{\delta}(p)=\nu \cdot\left|\nabla U_{1, \delta}(\nu p)\right|$, where $\nu>0$ is chosen such that $\nu p \in \Gamma_{1}$. In the present notation, Theorem 1 implies that

$$
K_{2, \delta}-K_{1, \delta} \geqslant\left|\Omega_{2} \backslash \Omega_{1}\right|_{\delta}-\left|\Omega_{1} \backslash \Omega_{2}\right|_{\delta}
$$

for all $0<\delta<\delta_{0}$, where ||$_{\delta}$ is the weighted area defined by the weight function $\left(a_{\delta}(p)\right)^{2}$. 
We have from the definitions that

$$
U_{i}(p)=U_{i, \delta}(p) \cdot\left(-(1 / 2 \pi) \operatorname{Ln}(\delta)+W_{i}(0)+o(\delta)\right)
$$

uniformly on $\Gamma_{\delta}$, where $o(\delta) \rightarrow 0$ as $\delta \rightarrow 0$. Since $U_{i}(p)=U_{i, \delta}(p)=0$ on $\Gamma_{i}$, it follows from the maximum principle that (7) holds uniformly on $\operatorname{closure}\left(\Omega_{i, \delta}\right), i=1,2$. It follows easily that

$$
\left|\nabla U_{i}(p)\right|=\left|\nabla U_{i, \delta}(p)\right| \cdot\left(-(1 / 2 \pi) \operatorname{Ln}(\delta)+W_{i}(0)+o(\delta)\right)
$$

uniformly on $\Gamma_{i}, i=1,2$. Therefore

$$
a(p)=a_{\delta}(p) \cdot\left(-(1 / 2 \pi) \operatorname{Ln}(\delta)+W_{1}(0)+o(\delta)\right)
$$

uniformly on $\left(\Omega_{1} \backslash \Omega_{2}\right) \cup\left(\Omega_{2} \backslash \Omega_{1}\right)$, and

$$
\begin{aligned}
\left|\Omega_{1} \backslash \Omega_{2}\right|-\left|\Omega_{2} \backslash \Omega_{1}\right|= & \left(\left|\Omega_{1} \backslash \Omega_{2}\right|_{\delta}-\left|\Omega_{2} \backslash \Omega_{1}\right|_{\delta}\right) \\
& \cdot\left(-(1 / 2 \pi) \operatorname{Ln}(\delta)+W_{1}(0)+o(\delta)\right)^{2} .
\end{aligned}
$$

By integrating (8) on $\Gamma_{i}$, one obtains

$$
1=K_{i, \delta} \cdot\left(-(1 / 2 \pi) \operatorname{Ln}(\delta)+W_{i}(0)+o(\delta)\right), \quad i=1,2
$$

Substitution of (10) and (11) into (6) yields, after a brief computation, that

$$
\begin{aligned}
W_{1}(0)-W_{2}(0) \geqslant & \left(\frac{-\operatorname{Ln}(\delta)+2 \pi W_{2}(0)}{-\operatorname{Ln}(\delta)+2 \pi W_{1}(0)}\right) \\
& \cdot\left(\left|\Omega_{1} \backslash \Omega_{2}\right|-\left|\Omega_{2} \backslash \Omega_{1}\right|\right)+o(\delta) .
\end{aligned}
$$

The inequality (4) follows in the limit as $\delta \rightarrow 0$. At this point the additional assumption that $\Gamma_{1}$ and $\Gamma_{2}$ have bounded curvature can be eliminated by the method used to eliminate the same restriction in the proofs of [1, Theorems 6 and 8].

LEMMA 5. Theorems 2 and 4 are equivalent.

Proof. For $0<\delta<1$, define $\gamma_{i, \delta}=\left\{p \in \Omega_{i}: U_{i}(p)=-(1 / 2 \pi) \operatorname{Ln}(\delta)\right\}$, $i=1$, 2. Then $|p|=\delta \cdot\left(\exp \left(2 \pi W_{i}(0)\right)+o(\delta)\right)$ uniformly over all $p \in \gamma_{i, \delta}$, $i=1,2$. If we set $\Omega=\Omega_{1}$ and $\bar{\Omega}=\Omega_{2}$ (under the equivalence $z=x+i y=$ $(x, y)=p)$ then $F^{-1}(\bar{F}(z))$ maps $\gamma_{2, \delta}$ onto $\gamma_{1, \delta}$. Therefore

$$
\begin{aligned}
\frac{\left|\bar{F}^{\prime}(0)\right|}{\left|F^{\prime}(0)\right|} & =\operatorname{limit}_{\delta \rightarrow 0}\left(\frac{\exp \left(2 \pi W_{1}(0)\right)+o(\delta)}{\exp \left(2 \pi W_{2}(0)\right)+o(\delta)}\right) \\
& =\exp \left(2 \pi\left(W_{1}(0)-W_{2}(0)\right)\right) .
\end{aligned}
$$

The equivalence of Theorems 2 and 4 follows from (13) and the fact that $2 \pi \cdot\left|\nabla U_{1}(p)\right|=\left|F^{\prime}(z)\right|$ for $p=z \in \Gamma_{1}=\Gamma$.

\section{REFERENCES}

1. A. Acker, Heat flow inequalities with applications to heat flow optimization problems, SIAM J. Math. Anal. (to appear). 
2. A free boundary optimiation problem, SIAM J. Math. Anal. (to appear). Abstract; Notices Amer. Math. Soc. 23 (1976), p. A-645.

3. , Isoperimetric inequalities involving heat flow under linear radiation conditions, Proc. Amer. Math. Soc. (to appear).

4. L. Bieberbach, Zur Theorie und Praxis der konformen Abbildungen, Rend. Circ. Mat. Palermo 38 (1914), pp. 98-112.

5. T. Carleman, Uber ein Minimalproblem der mathematischen Physik, Math. Z. 1 (1918), pp. $208-212$.

6. L. E. Payne, Isoperimetric inequalities and their applications, SIAM Rev. 9 (1967), pp. 453-488.

7. G. Szegö, Über einige Extremalaufgaben der Potentialtheorie, Math. Z. 31 (1930), pp. 583-593.

MATHEMATISChe INSTTUUt I, UNIVERSTT̈̈t KARLSRUHE (TH), 75 KARLSRUHB 1, ENGLERSTRASSE 2, Postrach 6380, Federal Repubuc of Germany 